\title{
MONITORING OF CHLAMYDIA TRACHOMATIS GENITOURINARY INFECTION IN WOMEN - ANALYTICAL COMPARATIVE STUDY USING PUBLIC HEALTH RECORDS FROM TWO BALKAN COUNTRIES
}

\author{
Dragutin Arsić1, Dragan R. Milovanović ${ }^{2}$ Arbresa Bedzeti Ferati ${ }^{3}$, Zoran Prokić4, Vladan Vlajković1, Kenan \\ Ferati ${ }^{3}$, Jovana Arsić ${ }^{5}$ \\ ${ }^{1}$ Institute for Public Health Ćuprija Pomoravlje, Ćuprija, Serbia \\ ${ }^{2}$ Clinical Centre Kragujevac, Faculty of Medical Sciences, University of Kragujevac, Kragujevac, Serbia \\ ${ }^{3}$ Faculty of Medicine, University of Tetovo, Tetovo, FYR of Macedonia \\ ${ }^{4}$ Polyclinic Alba, Paracin, Serbia \\ ${ }^{5}$ Faculty of Business-Civil Security, International University of Brčko District, Brčko, Bosnia and Herzegovina
}

\section{SUMMARY}

Aim: This study investigated the cumulative incidence of Chlamydia trachomatis infection in women treated in gynaecology departments of healthcare facilities in two towns in Serbia and one town in the Former Yugoslav Republic (FYR) of Macedonia, including their medical records in public health reports.

Methods: A cross-sectional observational research design with retrospective data collection during a five-year period (2008-2012) originated from women treated as in- and out-patients. The data included the results of cervical and urethral swab testing on Chlamydia trachomatis infection and women's gynaecological diagnoses in Pomoravlje County (the Institute of Public Health Cuprija "Pomoravlje" in Cuprija, Alba outpatient clinic, Paracin) and in Skopje (Clinic for Obstetrics and Gynaecology, "Mikrolab" laboratory).

Results: The incidence of positive ELISA assay from samples from the Institute of Public Health "Pomoravlje" Cuprija and polyclinic Alba was $6.5 \%$ and $12.5 \%$, respectively $(p<0.01)$. The incidence of positive DFA test from samples from the Clinic for Obstetrics and Gynaecology, Skopje and "Mikrolab" laboratory was $18.8 \%$ and $15.2 \%$, respectively $(p=0.20)$. In Pomoravlje County and in Skopje $7.5 \%$ and $17.6 \%$ of urethral swab samples were positive for Chlamydia trachomatis, respectively $(p<0.01)$. The rate of microbiological samples tested for Chlamydia trachomatis in Pomoravlje County and Skopje was $48.4 \%$ and $21.2 \%$, respectively $(p<0.01)$. One-year incidences of Infectio sexuales chlamydiales (A56) from 2007-2011 were significantly different among three data settings relating to Serbia, the FYR of Macedonia and Pomoravlje County ( $p<0.001)$.

Conclusions: The incidence of Chlamydia trachomatis positive cervical and urethral swabs in our study were highly variable between countries and within individual hospitals and caused by many factors.

Key words: chlamydia trachomatis, female urogenital diseases, enzyme-linked immunosorbent assay, fluorescent antibody technique direct, infertility

Address for correspondence: D. Arsić, Institute for Public Health Ćuprija Pomoravlje, Miodraga Novakovica 78; 35230 Ćuprija, Serbia. E-mail: vitaaars@yahoo.com

http://dx.doi.org/10.21101/cejph.a4088

\section{INTRODUCTION}

The infection caused by Chlamydia trachomatis is one of the most common sexually transmitted diseases. It is estimated that during one year over 90 million people around the world get infected with this microorganism, with about one million new cases in the United States alone $(1,2)$. Chlamydia trachomatis causes several different genitourinary infections and, unsurprisingly, becomes a common cause of infertility in women (3). Fallopian tube infection itself plays a significant role in etiopathogenesis of infertility, and consequently pelvic inflammatory disease as its complication, which further increases infertility risk at least several times. For example, it is estimated that untreated Chlamydia trachomatis infection will lead to complications in form of pelvic inflammatory disease in many women, out of which a significant number of them become infertile. An additional problem of chlamydial infection is the association with ectopic pregnancy. Epidemiological evidence for it is still varying and setting-dependent, but there are strong data that suggest that the presence of biological markers of chlamydial infection represent a reliable indicator for the risk of ectopic pregnancy, as well $(4,5)$. Therefore, timely diagnosis and therapy of this disease are of crucial importance.

Nevertheless, it is surprising that there are few published studies dealing with epidemiological data of Chlamydia trachomatis infection in general population, both in Serbia and the neighbouring countries, throughout the period of socioeconomic transition. Except for a few recent reports, publicly available data on chlamydial 
infection prevalence among infertile women in the Balkan countries are scarce $(6,7)$. The epidemiological data are particularly scant for the subpopulation at risk, such as teenagers and young adults, promiscuous persons and women with previous diseases of genital system such as cervical ectopy or other chronic infections. Little is known about the prevalence of other predisposing factors in population affected with chlamydial infection. Such factors include, for example, early sexual activities, the nature of sex life, socioeconomic status, or the type of contraception methods used. Finally, research papers investigating incidence data are sparse.

The studies performed in Serbia suggested a high prevalence of Chlamydia trachomatis genitourinary infection in females. In a study that was conducted in Novi Sad, chlamydial infection was confirmed in $29.51 \%$ of adult women aged $19-67$ years (8). A previous research conducted in Belgrade reported the prevalence of Chlamydia trachomatis genital infection of $30.3 \%$ among sexually active female adolescents aged 19 years, who visited the National Institute for Mother and Child Health (9). Other studies were aimed at specific groups, such as women with premature delivery (10) or microorganisms closely related to Chlamydia trachomatis such as Ureaplasma (11). In general, the results mentioned above, as well as other similar studies, could suffer from selection bias since their methods were not based on epidemiological backgrounds.

Similarly, it could also be true for surrounding countries, which were constituents of the former Yugoslavia. The survey conducted in Montenegro found the prevalence of chlamydial infection of $4.17 \%$ in the general population but for the subgroup of women aged 26-35 years the frequency was ten times greater (47.8\%) (12). In the Former Yugoslav Republic (FYR) of Macedonia, the screening on Chlamydia trachomatis in group of women and men selected from the general population found this infection in $1.6 \%$ (95\% confidence interval $0.8-2.4 \%$ ) among persons without genitourinary infection symptoms and in $6.2 \%$ (3.1-9.3\%) of symptomatic subgroup (13). The additional, small-size study in this country reported $24.3 \%$ prevalence of Chlamydia trachomatis presence in genitourinary system in woman (14).

Countries of Western Balkans (Southeast Europe) share, to a great extent, similar healthcare environment and socioeconomic characteristics typical for societies in transition (15). That is why the joint initiatives dealing with both prevention and treatment of diseases including infectious diseases are getting more and more important $(16,17)$. The first step in this direction is the evaluation of population health based on the valid epidemiological data. In this regard, the aim of this comparative study was to examine the cumulative incidence of Chlamydia trachomatis infection in the population of women who are treated in gynaecology departments of healthcare facilities in two towns in Serbia and the FYR of Macedonia including their medical records in the public health reports.

\section{MATERIAL AND METHODS}

The study was designed as a cross-sectional observational research with retrospective data collection (18). A cross-section investigation covered a five-year period (2008-2012). Cumu- lated data related to women treated as in- and out-patients were entered into the study database, including the results of cervical and urethral swab testing for Chlamydia trachomatis infection and women's gynaecological diagnoses.

The retrospective approach to the number and types of swabs as well as data about diagnoses and their number reported as the first episode was performed according to the previously recommended epidemiological method (19). In particular, the diagnosis of infertility (N97) was identified as well as sexually transmitted infection of Chlamydia trachomatis (A56) according to the disease classification system of the World Health Organization (20). The study included data of two independent cohorts of women from Pomoravlje County (Serbia) and Skopje, the capital of Macedonia. All data were collected in a cumulative manner, based on summary epidemiological reports and no patient personal information was available to the researchers. The study approval was obtained from the management of the institutions (21).

Data from Pomoravlje County were collected from two checkpoints: the archive of the Institute of Public Health Cuprija "Pomoravlje" in Cuprija (microbiological laboratory, vital statistics and morbidity) and Alba - the privately-owned gynaecology and obstetrics outpatient clinic in Paracin. The data from the Institute concerning women treated mostly in public healthcare institutions in Pomoravlje County were collected within the national healthcare system, such as hospital outpatient departments in three major cities (Cuprija, Paracin and Jagodina) and partly from other ambulatory healthcare institutions in the area, including the admission department of the Institute itself. For the detection of Chlamydia trachomatis antigen in patients' cervical and urethral swabs both institutions in Cuprija used the enzyme-linked immunosorbent assay (ELISA) with the sensitivity of $65 \%$ and specificity near $100 \%$ (22).

Data from Skopje included the archives from two medical centres, the Clinic of Obstetrics and Gynaecology, Faculty of Medicine, University of Skopje and microbiological laboratory "Mikrolab", Skopje for the five-year period (2008-2012) (21). The archive of the clinic, in general, covered the information on women treated in both the hospital wards and outpatient department, while the data from "Mikrolab" laboratory referred exclusively to the patients who were treated in ambulatory settings. The analysis of patients' cervical and urethral swabs for the presence of Chlamydia trachomatis antigen was performed using direct fluorescent antibody assay (DFA) with the sensitivity of $88 \%$ and specificity near $100 \%$ (23).

Statistical analysis included methods of descriptive statistics and hypothesis testing using $\chi^{2}$-test and one-way analysis of variance-ANOVA (24). The number of episodes of appropriate diagnosis and diagnosis of all gynaecological disorders, infertility (N97) and sexually transmitted disease caused by Chlamydia trachomatis (A56) is presented in the form of cumulative incidence for a five-year period, in accordance with the appropriate epidemiological methods $(18,19)$. Incidence data for the first five-year period (2007-2011) were standardized per 100,000 population based on source morbidity data published within the appropriate annual statistical publications of national and regional public health institutes in Serbia* and in the FYR of Macedonia**, including another appropriate publication, as well (Table 1) (21).

\footnotetext{
*The Health Statistical Yearbook of Republic of Serbia, Institute of Public Health of Serbia "Dr Milan Jovanovic Batut", Belgrade, available at www.batut.org.rs/index.php?content=77; Health statistical yearbook, available from the Institute for Public Health Cuprija "Pomoravlje", Cuprija **The Health Map of the FYR of Macedonia, available from the Institute of Public Health of the FYR of Macedonia, Skopje, www.iph.mk
} 


\section{RESULTS}

Pomoravlje County data were represented with a total of 1,400 cervical smears of which 1,240 were tested at the Institute of Public Health "Pomoravlje" Cuprija and 160 were tested in the Alba Clinic, Cuprija. Of these, 100 smears were positive for Chlamydia trachomatis, i.e. $7.1 \%$ of all samples. The incidence of positive samples in the two institutions was significantly different, $6.5 \%$ in the Institute and $12.5 \%$ in Alba Clinic $\left(\chi^{2}=7.8\right.$, $\mathrm{df}=1, \mathrm{p}<0.01)$. The largest number of swabs were collected from outpatient subjects, only 25 swabs originated from women treated at three hospitals in Pomoravlje county, and 5 (20\%) of them were positive for Chlamydia trachomatis (Fig. 1).

In Skopje, data was based on examination of 715 cervical smears, of which 320 were collected in the ambulatory patients of the Clinic of Gynaecology and Obstetrics, and 395 in the "Mikrolab" laboratory. Of these, a total of 120 swabs were positive for Chlamydia trachomatis, which makes $16.8 \%$ of all samples. The incidence of positive samples in the Department of Obstetrics and Gynaecology and "Mikrolab" laboratory was $18.8 \%$ and $15.2 \%$, respectively, the difference was not statistically significant $\left(\chi^{2}=\right.$ 1.6, $\mathrm{df}=1, \mathrm{p}=0.20$ ). All samples taken at the Department of Gynaecology and Obstetrics originated from ambulatory patients since the data on samples of women treated at the hospital ward were not available (Fig. 1).

There was a statistically significant difference in the total number of positive findings in cervical swab samples taken in Pomoravlje County and Skopje $(\mathrm{p}<0.01)$. Although the number of urethral swabs in these locations was much smaller, there was a statistically significant difference between them in the frequency of swabs positive for Chlamydia trachomatis. Thus, in Pomoravlje County, out of 200 samples taken, 15 urethral swabs were positive (7.5\%), while in Skopje, from 320 urethral swab samples infection with Chlamydia trachomatis was identified in $60(17.6 \%)\left(\chi^{2}=\right.$ 10.8, $\mathrm{df}=1, \mathrm{p}<0.01)$.

During the five year period 410 cases of infertility (N97) were recorded at the wards of three hospitals in Pomoravlje county, which accounted for $34.3 \%$ of all cases of gynaecological diseases treated in these departments $(n=1,195)$. In the same period, the number of infertility cases admitted in the hospital ward of the Clinic of Obstetrics and Gynaecology, Skopje accounted for $63.6 \%$ of all gynaecological diseases treated there $(1,156$ of 1,817$)$. The difference in incidence of infertility treated in monitored wards in Pomoravlje County and Skopje was statistically significant $\left(\chi^{2}=249.3, \mathrm{df}=1, \mathrm{p}<0.01\right)$. On the other hand, the data on the exact incidence of cases of infertility in women treated as outpatients were less reliable. Consequently, it was found that during a five-year period at the General Hospital in Cuprija the incidence of infertility was 482 cases, in the Alba polyclinic 218 cases, and in the Department of Gynaecology and Obstetrics in Skopje 1,155 cases. A separate analysis of the incidence of infertility cases in ambulatory and hospital settings both in Pomoravlje county and Skopje was not possible due to methodological constraints.

The analysis of microbiological samples tested for Chlamydia trachomatis in relation to a five-year incidence of all gynaeco-

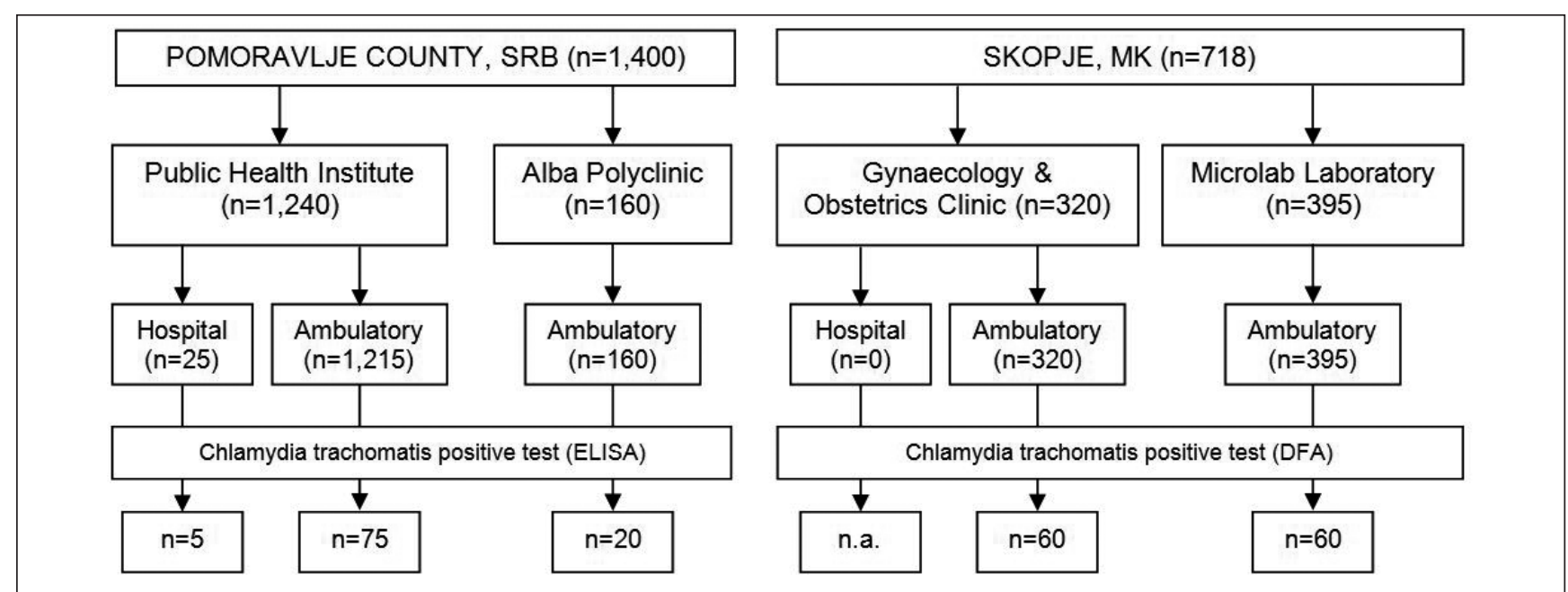

Fig. 1. Prevalence of cervical and urethral swabs positive for Chlamydia trachomatis presence in the 2008-2012 period in Pomoravlje County and Skopje.

Table 1. The Infectio Morbiditet sexuales chlamydiales (A56) for the period from 2007-2011 in Serbia, FYR of Macedonia and Pomoravlje County (calculated for 100,000 inhabitants)*

\begin{tabular}{|l|c|c|c|}
\hline Year & Serbia & FYR of Macedonia & Pomoravlje County \\
\hline 2007 & 21.0 & 1.3 & 1.4 \\
\hline 2008 & 31.6 & 2.5 & 3.9 \\
\hline 2009 & 26.8 & 1.8 & 0.0 \\
\hline 2010 & 15.7 & 1.8 & 2.2 \\
\hline 2011 & 15.1 & 2.3 & 1.4 \\
\hline
\end{tabular}


logical diseases indicates a significant difference between the monitored institutions in Pomoravlje County and Skopje. While the rate of sampling was $48.4 \%$ in Cuprija $(1,400$ samples from a total of 2,895 cases $)$, it was only $21.2 \%$ (715 of 3,368) in Skopje, resulting in a statistically significant difference $(\mathrm{p}<0.01)$. Regarding the fact that the available source records rarely match a microbiological sample with corresponding gynaecological diagnosis the detailed analysis of the relationship between laboratory findings and gynaecological diseases (including infertility) could not be carried out, especially given the incompleteness of data for women treated in the outpatient settings.

Data on one-year incidence Infectio sexuales chlamydiales (A56) for the period 2007-2011 are given in Table 1. There is a highly significant statistical difference in the incidence among the three groups (ANOVA, $\mathrm{F}=38.3, \mathrm{df}=2, \mathrm{p}<0.001$ ) due to very high values for the entire territory of Serbia compared to Pomoravlje County and territory of the FYR of Macedonia.

\section{DISCUSSION}

The incidence of Chlamydia trachomatis positive cervical and urethral swabs in our study was highly variable, both between the countries and within individual hospitals, ranging from 6.5 to $16.8 \%$. This was, in comparison to other similar studies conducted in Serbia, lower by about 3-5 times $(8,9)$. On the other hand, compared to other studies carried out in the FYR of Macedonia, our results are either higher for more than two times or lower for about a quarter to a third $(13,14)$. The data from Montenegro also show considerable variability and results for the general population are closer to those in Pomoravlje County than to the data from Skopje (12). In contrast to few studies from the Western Balkans, the current world literature is very extensive but also indicates considerable variability in the prevalence of Chlamydia trachomatis infection, depending on the study settings $(3,25)$. Similarly, decades ago Chlamydia trachomatis presence was reported in $1-7 \%$ of healthy males compared to $5-20 \%$ of asymptomatic females attending health facilities (26).

There are probably two main reasons for this variability that make direct comparison of data from studies conducted in different countries and even within different areas and/or institutions in the same country difficult. The first relates to the enrollment of subjects with very different characteristics, which could lead to the selection bias. The incidence of chlamydial infection was the lowest in the general population of men and women to be gradually increased according to the aggregation of certain specificities, such as the presence of symptoms or overt disease, female gender and specific age groups, especially sexually active adolescents and young people with high-risk behaviour. One of four healthcare facilities in our study was a small, ambulatory, privately owned facility specialized in women's health. Therefore, it is very likely that the high incidence of chlamydial test positivity in that unit resulted from both the patient selection bias and the smaller number of swab samples collected compared to other three departments. In the above-mentioned studies, subpopulations with heterogeneous characteristics were included, so it is quite conceivable that their results were considerably different.

Another reason for this variability comes from differences in diagnostic standards. In our study, the data from two countries were examined by two different laboratory methods (ELISA, DFA) with different diagnostic efficacy (particularly sensitivity) resulting in difference in frequency of positive tests for Chlamydia trachomatis infection. Additionally, there was a possibility of non-compliance of practicing physicians with the established diagnostic algorithms. For example, the US Center for Disease Control and Prevention defines a number of steps in diagnosis and treatment of Chlamydia trachomatis infection, with the special emphasis on vulnerable populations (27). However, it is very likely that consistent implementations of such or similar guidelines in a routine clinical practice concerning sexually transmitted diseases are highly variable (28).

In our study, we performed a secondary analysis dealing with infertility data since Chlamydia trachomatis infection was important causative factor of that condition (29). However, the discrepancy has been noticed between available epidemiological data regarding two data sets: the first, the reported gynaecological diagnosis and, the second, distribution of swabs taken and tested for the presence of chlamydial infection. While the largest number of women with infertility was treated in hospital wards in the Pomoravlje County and Skopje, the great majority of swabs (from cervix) were collected from women treated in outpatient facilities. Additionally, cumulative data for the reported gynaecological diseases from outpatient facilities was missing so that it was not possible to calculate the prevalence of infertility in comparison to all other gynaecological diseases in women treated during the study period.

Consequently, we were unable to calculate the accurate cumulative incidence of chlamydial infection in women with infertility. Both in Pomoravlje County and in Skopje the public health documentation used in our study was not based entirely on individual health records (30). Therefore, different women, due to multiple hospital and outpatient treatment courses, differently participated in the collected data sets. Only indirectly, our results can be compared with similar studies. For example, in Serbia, in women with primary infertility, Chlamydia trachomatis antigen was detected in $15.06 \%$ of subjects and in healthy females only in $3.50 \%$ of subjects (31). Other researchers found seropositivity against Chlamydia trachomatis in $64.5 \%$ of males with some fertility problems, but the exact prevalence showed double difference depending on the underlying cause (7). The researchers from Romania reported that the prevalence of Chlamydia trachomatis antigen and Chlamydia trachomatis IgG antibodies found in infertile women was $0.8 \%$ and $15.20 \%$, respectively, but the positivity for the latter was found in $3.33 \%$ of healthy controls (6). In Slovakia, the researchers have recently found the point prevalence of Chlamydia trachomatis infection in $6.5 \%$ of investigated subjects with slight difference between people from the majority population and those from the ethnic minority group $(5.3 \%$ vs. $7.2 \%)(32)$. On the other hand, chlamydial infection was found in $5.7 \%$ of women with infertility in Scotland (25) and in $18.6 \%$ of infertile women in India where researchers used a highly sensitive diagnostic test (3). Even among couples treated for infertility the frequency of detection of Chlamydia infection varied from $1 \%$ to $13 \%(29)$. All these figures roughly correspond to the previously observed variability in incidence of this infection for all our patients, in both geographic areas.

In our study, data from routine morbidity monitoring system of the two countries, and even between different regions or health 
facilities were extremely heterogeneous and often incomplete or even absent. The information was documented mainly by cumulative approach, without other sufficient details such as sociodemographic and epidemiological data on the patient, the course of disease and comorbidities. Consequently, we were unable to analyze the relationships between the patients' characteristics and the swab sample results concerning either the interindividual (e.g. differences of positivity of cervical and urethral swabs) or the intraindividual issues (e.g. age-specific incidence rate). In addition, stratification of the data according to the type of healthcare institutions, their sub-units (e.g., hospital wards, outpatient departments, offices) and place in the network of national healthcare system were often incomplete or absent. Finally, the quality of implementation of existing guidelines in this area was also unknown. Therefore, it is not surprising that the trend of mortality due to Chlamydia trachomatis sexual infections in our study significantly differed between the two countries, and the differences were found not only at the national but also at regional levels. As the organization and implementation of systematic monitoring of morbidity is extremely heterogeneous, it constitutes an aggravating factor for all health improvement activities.

The need for harmonization of the monitoring system in public health among European countries has already been recognized, including the region of Southeast Europe or Balkans $(33,34)$. This is particularly important for infectious diseases such as viral hepatitis, but also for other public health issues (35). In this regard, standardized collection and analysis of original data, with special emphasis on the individual records of patients (e.g. electronic record) is of crucial importance in order to overcome current obstacles (30). The latest findings that the health status of the population in Eastern European countries, loaded with numerous economic and health problems, is less favourable than in developed European regions only highlights the urgent need to implement effective strategies into national healthcare systems (36). The results of our study provide new evidence in this regard and support the previous observation.

In conclusion, infections with Chlamydia trachomatis represent a significant health problem for women in Serbia and the FYR of Macedonia, but there are difficulties in epidemiological data availability in routine medical records that might be used for routine epidemiological monitoring in public healthcare system. There is a need for the harmonization of system on supranational level, and future studies should provide evidence for optimal strategies.

\section{Acknowledgements}

DRM thanks to the Ministry of Education, Science and Technological Development of the Republic of Serbia for partial support through the research grant No. 175007. Authors thank to Ms Nevena Simsic for the language editing.

\section{Conflict of Interests}

None declared

\section{Adherence to Ethical Recommendations}

The study design, conduction and reporting adhere to local and international ethical recommendations for medical research.

\section{REFERENCES}

1. Norman J. Epidemiology of female genital Chlamydia trachomatis infections. Best Pract Res Clin Obstet Gynaecol. 2002 Dec;16(6):775-87.

2. Mishori R, McClaskey EL, WinklerPrins VJ. Chlamydia trachomatis infections: screening, diagnosis, and management. Am Fam Physician. 2012 Dec 15;86(12):1127-32.

3. Mania-Pramanik J, Kerkar S, Sonawane S, Mehta P, Salvi V. Current Chlamydia trachomatis infection, a major cause of infertility. J Reprod Infertil. 2012 Oct;13(4):204-10.

4. Bakken IJ. Chlamydia trachomatis and ectopic pregnancy: recent epidemiological findings. Curr Opin Infect Dis. 2008 Feb;21(1):77-82.

5. Daponte A, Pournaras S, Deligeoroglou E, Skentou H, Messinis IE. Serum interleukin-1 $\beta$, interleukin- 8 and anti-heat shock 60 Chlamydia trachomatis antibodies as markers of ectopic pregnancy. J Reprod Immunol. 2012 Mar;93(2):102-8.

6. Mareş M, Socolov D, Doroftei B, Botezatu A, Goia CD. The prevalence of some bacterial markers in female patients undergoing an initial infertility evaluation in north-east Romania. Roum Arch Microbiol Immunol. 2009 Jul-Sep;68(3):171-4.

7. Arsovic A, Nikolov A, Sazdanovic P, Popovic S, Baskic D. Prevalence and diagnostic significance of specific IgA and anti-heat shock protein 60 Chlamydia trachomatis antibodies in subfertile women. Eur J Clin Microbiol Infect Dis. 2014 May;33(5):761-6.

8. Jerant-Patić V, Milosević V, Kozarev G. Chlamydia trachomatis in women. Med Pregl. 2009 Jan-Feb;62(1-2):7-12. (In Serbian.)

9. Sedlecki K, Markovic M, Rajic G. Risk factors for Clamydia infections of the genital organs in adolescent females. Srp Arh Celok Lek. 2001 Jul-Aug;129(7-8):169-74. (In Serbian.)

10. Bogavac M, Aleksić S, Radulović A. Chlamydia trachomatis-a possible cause of premature labor. Med Pregl. 2002 Mar-Apr;55(3-4):146-8. (In Croatian.)

11. Randelović G, Kocić B, Miljković-Selimović B, Mladenović-Antić S, Stojanović P, Stefanović M. High-density cervical Ureaplasma urealyticum colonization in pregnant women as a risk factor for premature rupture of membranes. Vojnosanit Pregl. 2006 Aug;63(8):737-41. (In Serbian.)

12. Vuksanovic V, Bujko M. Genital infection caused by chlamydia trachomatis in females and males in different age cycle. Glasnik Zavoda Zastitu Zdravlja Srbije. 2000;74(1-4):33-6. (In Serbian.)

13. Spasovski MS, Simjanovska LJ, Taleski V, Petrova N, Lazetic L, Popeska Z, et al. Screening of Chlamydia trachomatis urogenital infections among the male and female population of the Republic of Macedonia. J Eur Acad Dermatol Venereol. 2005 Jul;19(4):427-30.

14. Tanturovski D, Grdanoska T, Petrovska M, Stojovski M, Tanturovski M. Presence of Chlamydia trachomatis in the women's urethra during a concomitant generic infection of cervix uteri. Maced J Med Sci. 2008 Sep;1(1):41-3.

15. Eikemo TA, Huisman M, Perlman F, Ringdal K. Educational health inequalities in former Yugoslavia: evidence from the South-East European Social Survey Project. Eur J Public Health. 2010 Dec;20(6):640-6.

16. Burazeri G, Laaser U, Bjegovic V, Georgieva L; Consortium for Public Health Collaboration in South Eastern Europe. Regional collaboration in public health training and research among countries of South Eastern Europe. Eur J Public Health. 2005 Feb;15(1):97-9.

17. Bino S, Cavaljuga S, Kunchev A, Lausevic D, Kaic B, Pistol A, et al. Southeastern European Health Network (SEEHN) Communicable Diseases Surveillance: a decade of bridging trust and collaboration. Emerg Health Threats J. 2013;6. doi: 10.3402/ehtj.v6i0.19950.

18. Jevtović I, Arsić D. Methods of scientific research. Belgrade: Faculty for Business and Industrial Management; 2013. (In Serbian.)

19. Instructions for recording data of interest to the whole country in health care, preparing and submitting reports-methodological matters. Nis: Institute for Documentation of Occupational Safety; 1978. (In Serbian.)

20. Ljubicic M, editor. International statistical classification of diseases and related health problems. 10th Revision. Belgrade: Institute of Public Health of Serbia „Dr Milan Jovanović-Batut“; 2010. (In Serbian.)

21. Ferati AB. The role of health management in research, microbiological diagnosis and prevention of genital infections caused by Chlamydia trachomatis in Macedonia [PhD dissertation]. Belgrade: European Center for Peace and Development of University of Piece; 2013. (In Serbian.)

22. Schmid G, Samuelson J, Rowley J. Prevalence and incidence of selected sexually transmitted infections. Chlamydia trachomatis, Neisseria gonorrhoeae, syphilis and Trichomonas vaginalis. Methods and results used by WHO to generate 2005 estimates. Geneva: World Health Organization; 2011. 
23. Sachdeva P, Patel AL, Sachdev D, Ali M, Mittal A, Saluja D. Comparison of an in-house PCR assay, direct fluorescence assay and the Roche AMPLICOR Chlamydia trachomatis kit for detection of C. trachomatis J Med Microbiol. 2009 Jul;58(Pt 7):867-73.

24. Jevtovic I. Medical statistics. Kragujevac: Medical Faculty University of Kragujevac; 2002. (In Serbian.)

25. Bhattacharya S, Porter M, Amalraj E, Templeton A, Hamilton M, Lee AJ, et al. The epidemiology of infertility in the North East of Scotland. Hum Reprod. 2009 Dec;24(12):3096-107.

26. Nongonococcal urethritis and other selected sexually transmitted diseases of public health importance. Report of a WHO Scientific Group. World Health Organ Tech Rep Ser. 1981;660:1-142.

27. Workowski KA, Berman S; Centers for Disease Control and Prevention (CDC). Sexually transmitted diseases treatment guidelines, 2010. MMWR Recomm Rep. 2010 Dec 17;59(RR-12):1-110.

28. Bangor-Jones RD. Sexual health in general practice: do practitioners comply with the sexually transmitted infections guidelines for management of suspected chlamydial infections? Int J STD AIDS. 2011 Sep;22(9):523-4.

29. Akande V, Turner C, Horner P, Horne A, Pacey A; British Fertility Society. Impact of Chlamydia trachomatis in the reproductive setting: British Fertility Society Guidelines for practice. Hum Fertil (Camb). 2010 Sep;13(3):115-25.

30. Kolsek M. Implementing electronic medical record in family practice in Slovenia and other former Yugoslav Republics: barriers and requirements. Srp Arh Celok Lek. 2009 Nov-Dec;137(11-12):664-9.
31. Indjic N, Vuckovic S, Andjelkovic Z, Veinovic J, Jovanovic M, Zivulovic S. Prevalence Chlamydia trachomatis and female infertility. PONS Med J. 2013 Sep;10(3):101-3. (In Serbian.)

32. Halánová M, Jarcuska P, Kalinová Z, Cáriková K, Oravcová J, Jarcuska P, et al.; HepaMeta Team. The prevalence of Chlamydia trachomatis in the population living in Roma settlements: a comparison with the majority population. Cent Eur J Public Health. 2014 Mar;22 Suppl:S32-6.

33. Hulth A, Viso AC. European institutes for disease prevention and control collaborate to improve public health surveillance. Euro Surveill. 2011 Apr 28;16(17). pii: 19851.

34. Marusic A. Peace through public health in southeast Europe? Lancet. 2002 Jan 5;359(9300):54.

35. Hatzakis A, Van Damme P, Alcorn K, Gore C, Benazzouz M, Berkane $\mathrm{S}$, et al. The state of hepatitis B and $\mathrm{C}$ in the Mediterranean and Balkan countries: report from a summit conference. J Viral Hepat. 2013 Aug;20 Suppl 2:1-20.

36. Mackenbach JP, Karanikolos M, McKee M. The unequal health of Europeans: successes and failures of policies. Lancet. $2013 \mathrm{Mar}$ 30;381(9872):1125-34.

Received September 3, 2014 Accepted in revised form November 11, 2015 